\title{
Myocardial infarction in a young man
}

\author{
N P Singh, S Anuradha, Piyush Ranjan, S N A Rizvi
}

A 31-year-old male smoker, presented with a six-hour history of sudden onset, severe, retrosternal pain, associated with profuse sweating. There was no past or family history of ischaemic heart disease. The patient had a history of 'swelling' all over his body for the past two years. There was no haematuria or oligo-anuria. He had been prescribed only diuretics for his swelling, which he was taking off and on. On admission, the patient was anxious, in pain, and diaphoretic. His heart rate was regular, 92 beats/min, blood pressure $160 / 100 \mathrm{mmHg}$ and he had pedal oedema. The chest and cardiovascular examination was normal. A standard 12-lead electrocardiogram (ECG) on admission is shown in figure 1 . The results of the laboratory investigations are summarised in the table. Echocardiography done on the fourth day revealed apical hypokinesia. Further investigations were deferred. Three months later, a coronary angiography demonstrated entirely normal coronary vessels. A kidney biopsy was done and the histology is depicted in figure 2 .
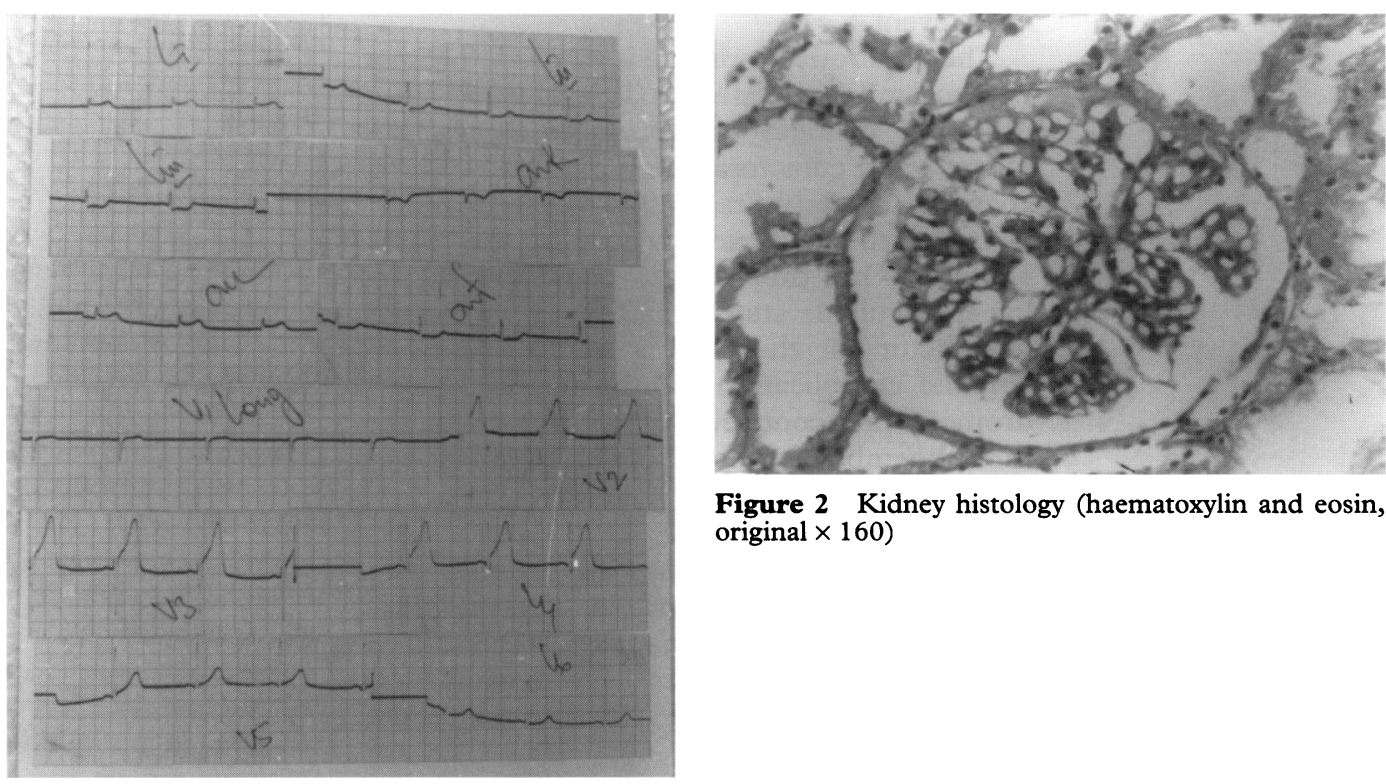

Figure 2 Kidney histology (haematoxylin and eosin original $\times 160$ )

Figure 1 Admission ECG

Table 1 Laboratory investigations

\begin{tabular}{llll}
\hline Investigation & Normal range & First admission & After 3 months \\
\hline Haemoglobin (g/dl) & $14-16$ & 14.2 & 13.0 \\
Serum albumin (g/dl) & $4-6$ & 1.6 & 1.9 \\
24-h urinary protein excretion (g) & - & 6.3 & 3.4 \\
Serum cholesterol (mg/dl) & $150-250$ & 336 & 351 \\
Serum triglycerides (mg/dl) & $100-200$ & 324 & 183.4 \\
Low-density lipoproteins (mg/dl) & $86-138$ & 223 & 173 \\
Apo B (mg/dl) & $63-114$ & - & 25.0 \\
Blood urea (mg/dl) & $20-40$ & 20.0 & 0.8 \\
Serum creatinine (mg/dl) & $0.8-1.6$ & 1.1 & 526.0 \\
Serum fibrinogen (mg/dl) & $150-400$ & - & 60.0 \\
Antithrombin III (\%) & $80-120$ & - & 126.0 \\
Protein C (\%) & $70-140$ & - & enhanced \\
Platelet adhesiveness to ADP and ristocetin & - & - & \\
\hline
\end{tabular}

Department of

Azad Medical College

\& Associated Lok

Nayak Hospital, New

Delhi - 110 002, India

N P Singh

S Anuradha

P Ranjan

S N A Rizvi

Correspondence to Dr NP Singh, 2104 Delhi

Administration Flats, Gulabi

Bagh, Delhi - 110 007, India

Accepted 20 August 1997

\section{Questions}

1 What is the primary diagnosis?

2 What is the complicating event and what is the underlying aetiology?

3 How will you manage such complications? 
Answers

QUESTION 1

The primary diagnosis is nephrotic syndrome as evidenced by proteinuria $(6.39 \mathrm{~g} / 24 \mathrm{~h})$, hyperlipidaemia (serum cholesterol $336 \mathrm{mg} / \mathrm{dl}$, triglycerides $324 \mathrm{mg} / \mathrm{dl}$ ), hypoalbuminaemia (serum albumin $1.6 \mathrm{~g} / \mathrm{dl}$ ) and oedema. The diffuse glomerular basement membrane thickening seen on kidney biopsy is characteristic of membranous glomerulonephritis, which is the underlying histological abnormality.

\section{QUESTION 2}

The patient suffered an acute anteroseptal myocardial infarction as the complicating event. This is evidenced by the ECG, raised levels of cardiac enzymes and echocardiography. Since no evidence of coronary artery disease was found on subsequent angiography, in-situ thrombosis of the coronary artery secondary to the hypercoagulable state of nephrotic syndrome, (demonstrated by hyperfibrinogenaemia, reduced antithrombin III levels and enhanced platelet aggregability) was the likely event.

The nephrotic syndrome is associated with a hypercoagulable state. ${ }^{1}$ It is associated with profound changes in fibrinogen levels, zymogens and cofactors, the fibrinolytic system, coagulation inhibitors and platelet functions, ${ }^{2}$ summarised in boxes 1 and 2. All these alterations are proposed to contribute to the hypercoagulable state, which is an important risk factor for the genesis of the thromboembolic complications of the nephrotic syndrome. Other contributory risk factors for the thromboembolic episodes are listed in box 3 .

QUESTION 3

It is generally agreed that all patients with nephrotic syndrome and a proven, lifethreatening thromboembolic complication should be anticoagulated. ${ }^{4}$ Anticoagulation should continue for at least six months after the

\begin{tabular}{|l|}
\hline Changes in the coagulation factors and \\
fibrinolytic system in patients with \\
nephrotic syndrome \\
\hline Increased \\
- fibrinogen antigen and activity \\
- factor VIII antigen and activity \\
- factor XIII antigen and activity \\
- combined factor II, VII, X, activity \\
- cofactor V \\
- alpha-2 macroglobulin \\
Reduced \\
- antithrombin III \\
- plasminogen \\
- factor XI \\
- factor XII \\
Areas of controversy \\
- protein S and C (up in some studies, down in \\
others) \\
\hline
\end{tabular}

Box 1
Changes in platelets in patients with nephrotic syndrome

- thrombocytosis

- increased adhesiveness

- increased ADP-induced rate and percentage of aggregation

- increased collagen-induced rate and percentage of aggregation

- shorter time for collagen-induced aggregation

- increased beta-thromboglobulin

Box 2

\section{Other risk factors contributing to the} hypercoagulable state

- diuretics: by causing volume depletion and haemoconcentration

- corticosteroids: by increasing factor VIII concentration

- hypoalbuminaemia: reducing antithrombin III levels, increasing fibrinogen levels, increasing platelet aggregation

- hyperlipidaemia: possibly enhancing platelet aggregation

- membranous nephropathy on histology

Box 3

proteinuria remits or until the serum albumin is lower than $2 \mathrm{~g} / \mathrm{dl}$. The role of prophylactic anticoagulation in preventing the occurrence of thrombotic events is not clearly established. While some researchers propose routine anticoagulation for all patients, especially those with membranous nephropathy, ${ }^{5}$ most physicians advocate an individualised approach. There is growing evidence for the role of thromboprophylaxis with antithrombotic agents like aspirin. ${ }^{2}$

In addition to the above, control of attendant risk factors like hypoalbuminaemia and proteinuria, hyperlipidaemia, hypertension and smoking, are an integral part of the management strategy.

\section{Discussion}

Thromboembolic episodes are wellrecognised, serious, and at times lifethreatening complications of the nephrotic syndrome. Although venous thromboses are more common, thrombotic occlusions of nearly all arteries have been described in the literature. ${ }^{6}$ Isolated coronary artery thrombosis in the nephrotic syndrome, in the absence of coronary artery disease, has rarely been documented. $^{7-9}$ The hypercoagulable state of the nephrotic syndrome is proposed to be responsible for these complications, as discussed earlier.

Normal coronary vessels on angiography in patients with a proven myocardial infarction is a well-recognised entity, especially in younger patients. Normal angiography in a patient with a myocardial infarction could be due to a number of causes, including a hypercoagulable 


\section{Learning points}

- nephrotic syndrome is a hypercoagulable state

- thrombotic episodes are frequent manifestations of the hypercoagulable state in nephrotic syndrome

- although less common than venous thrombosis, arterial thrombosis may be life-threatening

- normal coronary vessels may be seen in young patients with acute myocardial infarction

- a hypercoagulable state is a possible contributory factor to coronary artery thrombosis

- normal coronary angiography should prompt a search to rule out an underlying hypercoagulable state

Box 4

1 Kendall AG, Loohmann RC, Dossetor JB. Nephrotic syndrome: a hypercoagulable state. Arch Intern Med 1971;127:1021-7.

2 Llach F. Hypercoagulability, renal vein thrombosis and ther thrombotic complications of the nephrotic syndrome. Kidney Int 1985;28:429-39.

3 Fahal IH, McClelland P, Hay CRM, Bell GM. Arterial thrombosis in the nephrotic syndrome. Postgrad Med $\mathcal{f}$ 1994;70:905-9.

4 Cameron JS. Coaglation and thrombo embolic complications in the nephrotic syndrome. Adv Nephrol 1984;13:7514.

5 Bellomo R, Atkins RC. Membranous nephropathy and thromboembolism: is prophylatic anticoagulation warranted? Nephron 1993;63:249-54.

6 Kim HJ, Park CH, Kang CM, Park HC. Arterial thrombosis associated with the nephrotic syndrome - a case report and review (adult cases in English Literature). 7 Kor Med $S c i$ 1993;8:230-4. state. ${ }^{10}$ In all patients with a proven myocardial infarction and normal coronary vessels, a search should be made for any of these conditions.

\section{Final diagnosis}

Nephrotic syndrome complicated by acute anteroseptal myocardial infarction.

Keywords: nephrotic syndrome; myocardial infarction

7 Mukheriee AP, Toh BH, Chan GL, Lau KS, White JC. Vascular complications in the nephrotic syndrome: relationship to steroid therapy and accelerated thromboplastin generation. $B M \mathcal{F} 1970 ; 4: 273-6$

8 Fujimara O, Gulamhusein S. Acute myocardial infarction: thrombotic complications of nephrotic syndrome. Can $\mathcal{F}$ Cardiol 1987;3:267-9.

9 Lip G, Tean KN. Acute myocardial infarction: a rare complication of the thrombolic tendency in nephrotic syndrome. Br $\mathcal{f}$ Clin Pract 1994;48:218-20.

10 Pasternak RC, Braunwald E, Sobel BE. Acute myocardial infarction. In: Braunwald E, ed. Heart disease - a text book of cardiovascular medicine, vol 2, 4th edn. Philadelphia: WB Saunders, 1992; pp 1200-91.

\section{A dislocated finger}

\section{H Gerrand, P M Jarrett}

A 64-year-old man presented 12 hours after he fell onto his hand whilst intoxicated. X-Rays are shown in figure 1.

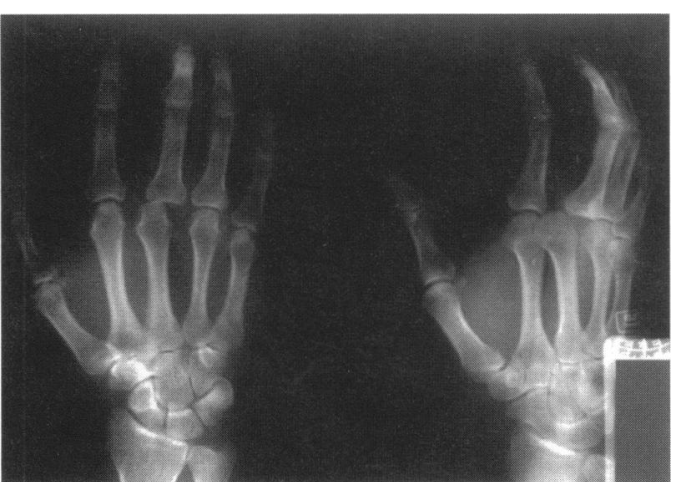

Figure $1 \mathrm{X}$-Rays of the patient's hand at presentation

\section{Questions}

1 What does the radiological appearance suggest?

2 What is the anatomy of this injury?

3 What is the correct treatment?
Department of

Orthopaedics, Western

Infirmary, Dumbarton

Road, Glasgow G11

6NT, UK

C H Gerrand

P M Jarrett 